\title{
Insulin Granule Segmentation in 3-D TEM Beta Cell Tomograms
}

\author{
David Nam ${ }^{1}$ \\ D.Nam@bristol.ac.uk \\ Judith Mantell ${ }^{2,3}$ \\ J.Mantell@bristol.ac.uk \\ David Bull ${ }^{1}$ \\ Dave.Bull@bristol.ac.uk
}

Paul Verkade $2,3,4 /$ shared last author

P.Verkade@bristol.ac.uk

Alin Achim ${ }^{1}$

Alin.Achim@bristol.ac.uk

\author{
${ }^{1}$ Visual Information Laboratory \\ University of Bristol, UK \\ ${ }^{2}$ Wolfson Bioimaging Facility \\ University of Bristol, UK \\ ${ }^{3}$ School of Biochemistry \\ University of Bristol, UK \\ ${ }^{4}$ School of Physiology and \\ Pharmacology \\ University of Bristol, UK
}

\begin{abstract}
We consider the problem of segmenting insulin granule cores and halos, from 3D transmission electron microscopy tomography data of beta cells. Due to the image acquisition procedure, granule segmentation is especially challenging. Along with the known difficulties in granule segmentation, such as image heterogeneity and complex cellular structure, other problems are posed with 3-D beta cell imaging. The granule membrane is partially or not at all visible; there is also poor contrast within the halos. Being able to quantify large amounts of volumetric data, will allow biologists to have a more accurate understanding of cellular mechanics than previously possible. We present an algorithm for segmentation of granule cores, and then extend to halos. We first detect cores using a localised region-based active contour on each 2-D slice, then perform a refining segmentation using dual region-based active surfaces. Within the algorithm we propose a novel active surface for granule halo segmentation. We also include a novel gradient based term, to attenuate dual surface attractions around the membrane. We demonstrate the effectiveness of our novel contribution, by comparing it to other stateof-the-art active surface models and then compare our results to manual segmentations. When compared to manual segmentations our approach produces a Dice's coefficient of 0.88 and 0.85 for core and halo segmentations respectively.
\end{abstract}

\section{Introduction}

Image informatics has become the rate-limiting factor in realising the full potential of dynamic cellular and molecular imaging studies [M]. Having large numbers of accurate quantitative data is integral to understanding cellular mechanisms. Insulin is the only hormone in mammals able to lower blood glucose levels and consequently it is vital for life [111], [17]. Typically, insulin granules are described as organelles containing a dense core, surrounded by a halo and an enclosing membrane Fig. 1. Not all granules show a surrounding halo; this 


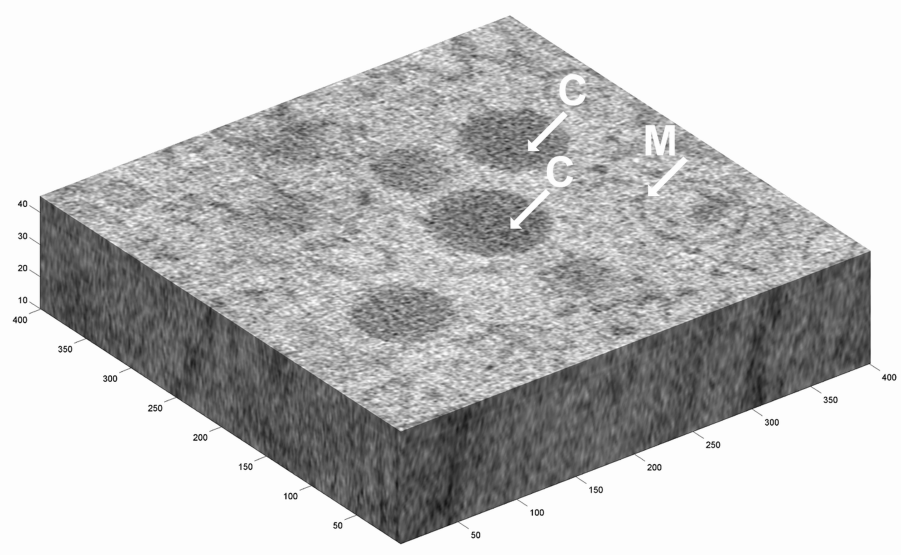

Figure 1: Cropped 3-D TEM data set of beta cell, $401 \times 401 \times 36$. The dark circles are the granule cores $C$. Note the weak membrane information $M$ presented in the image.

is very much dependent on the fixation procedure. Analysis of insulin granules in transmission electron microscopy (TEM) beta cell images is usually done manually. Currently, it is not feasible to analyse large numbers of 3-D TEM beta cell images - as manual segmentation is very time consuming. Recently, 3-D analysis was done on insulin granule images extrapolating 2-D data [7]. To get even more accurate data on insulin granule geometry and volume, automated granule segmentation methods need to be developed.

\subsection{3-D Electron Tomography Preparation}

3-D electron microscopy tomograms of beta cells are obtained using a similar procedure as for 2-D tomograms. Firstly, the sample has to be fixed using either a chemical or high pressure freezing fixation method. Conventional 2-D images of the sample are then obtained at different tilt orientations. The raw data is reconstructed into slices to form the 3-D image. Despite viewing the same type of cell as [1 3$]$ and [7], the 3-D beta cell images are quite different from their 2-D versions. The primary cause for this is the need for a thicker sample slice of the beta cell than in 2-D transmission electron microscopy but also the reconstruction algorithm plays a part. We used the most commonly used weighted back projection in the IMOD software package [11]. This leads to an image with poor contrast when compared to 2-D beta cell electron microscopy tomograms. The relatively poor contrast has the greatest effect on granule halos, which is demonstrated in Fig. 1.

\subsection{Microscopy Image Segmentation Methods}

Due to the complexity of beta cell transmission electron microscopy images, specific methods need to be developed in order to segment organelles of interest. In [y], mitochondria are segmented from serial block-face scanning electron microscopy images, using the random forest classifier and the level set active contour. Within [ $[$ ], cell nuclei are segmented in 3-D confocal microscopy images, using an algorithm based on the watershed method. 
[I] tackled the problem of mitochondria segmentation in 3-D focused ion beam scanning electron microscope images of brain cells, using an automated graph partitioning scheme. Having a dedicated method for 3-D granule segmentation can provide large amounts of accurate data - about insulin granules size and geometry - than previously possible. In recent works, the level set active contour segmentation method has proven successful in segmenting microscopy images: [13], [७], [6] and [4]. As a result of weak boundary information of granules in TEM images, region-based active contours [3], [I ], [19] and [19] have shown to be successful in granule core segmentation. Due to the decreased image contrast in 3-D TEM images, granule membranes are far less prominent (and in some cases unnoticeable) than their 2-D counterparts. In this case a region-based active contour would be applicable to solving this problem. Exploiting shape information can be beneficial when segmenting complex objects. In [IX] segmented contours are implicitly represented by applying principal component analysis on a collection of signed distance functions, representative of the training data. $[16]$ includes shape priors derived from training data, within a Bayesian level set framework.

We present an algorithm for serial segmentation of granule cores and membranes, utilising region-based active contours. An initial segmentation is first performed on each slice to get a good estimate of the granule core locations; a second refining segmentation is then done on each granule using an active surface, first for the core then the halo. We propose a novel boundary prior and gradient term, which we incorporate in a dual active surface, to address the challenges faced with halo segmentation. The remainder of the paper is described as follows: in Section 2 we describe our novel active contour, boundary prior and gradient term. We present our granule segmentation algorithm in Section 3. In Section 4 we validate our results by doing comparisons to manual segmentations and other state-of-the-art methods. We conclude in Section 5.

\section{Theoretical Preliminaries}

Active contour models have proven successful in image segmentation [14], [3] and [']. Geometric active contours represent a contour as the zero level set of a signed distance function. In particular, region-based active contours [3], [17] and [1.5] have been shown useful in microscopy image segmentation [6]. [14] proposed a generalised region-based scheme, similar to that of [3] but incorporates region variances. It is presented in a Bayesian framework, where region intensities are assumed to have a Gaussian distribution. 3-D TEM granules are challenging to segment for many reasons: they are comprised of many complex structures, they are also heterogeneous (between granules and within the image) and have weak membrane information. In some cases, granule halos are characterised as just a fuzzy, high pixel intensity band around the core. Alternatively, a halo may have similar intensities with the image background; in this case the only distinguishing feature is the homogeneity within the halo. This makes incorporating region variances advantageous in halo segmentation: a more homogeneous region will have less intensity variance. Given an image $I$ the conditional probability for intensities within its respective region, $i$-inside and outside the contouris defined as:

$$
p_{i}(I \mid C)=p_{\sigma_{i}, \mu_{i}}(I \mid C)=\frac{1}{\left(\sqrt{2 \pi} \sigma_{i}\right)} e^{-\frac{\left(I-\mu_{i}\right)^{2}}{2 \sigma_{i}^{2}}},
$$

where $\sigma_{i}$ and $\mu_{i}$, represent the standard deviation and mean intensities within region $i$. 


\subsection{Boundary Prior}

Presenting the level set active contour in a Bayesian framework provides an efficient way of incorporating shape priors into the energy term. During halo segmentation, it is important that the contour does not enter the core during its evolution. We present a level set boundary prior, to ensure that the halo segmentation always encapsulates the granule core. Our boundary prior has the advantage of not using training data but is instead based on the granule core segmentation. Given the probability of a region being partitioned by a contour $C$ as $p_{C_{\text {core }}}(C)$ the following relationship can be formed:

$$
p_{C_{\text {core }, i}}(C \mid I) \propto p_{i}(I \mid C) p_{C_{\text {core }}}(C) .
$$

In our algorithm we take $C_{c o r e}$ as the granule core, (which will be obtained previous to this step). We assume that all contours outside of the granule core are equally possible; therefore we define our boundary prior, $p_{C_{c o r e}}(C)$ to have the following probability distribution:

$$
\begin{aligned}
& p_{C_{\text {core }}}(C)=0-\text { if inside } C_{\text {core }}, \\
& p_{C_{\text {core }}}(C)=1-\text { if outside } C_{\text {core }} .
\end{aligned}
$$

Given a level set function $\phi$, we can define our boundary prior energy functional as follows:

$$
E_{\text {boun }}=-\log \left(1-H\left(\int\left(C_{\text {core }}-H_{\varepsilon}(\phi)\right) C_{\text {core }} d x\right)\right) .
$$

Where $H$ is the Heaviside function and $H_{\varepsilon}$ is a smoothed Heaviside function, with derivative $\delta_{\varepsilon}$ as shown in [3]. We can then define $M_{1}(\phi)=H_{\varepsilon}(\phi)$ and $M_{2}(\phi)=1-H_{\varepsilon}(\phi)$. In order to minimize the energy $E_{\text {boun }}$, we take the negative logarithm of the distribution $p_{C_{\text {core }}}(C)$, [1 $)$ ].

\subsection{Dual Bayesian Active Contour}

Despite the merits of incorporating image variances within a region-based active contour, more needs to be done to achieve accurate segmentations. We utilise the image energy functional presented in [1 ] ]:

$$
\begin{aligned}
& E(\phi)=\sum_{i=1}^{2} \frac{1}{2} \int\left(\log \left(\sigma_{i}^{2}\right)+\left(\frac{\left(I-\mu_{i}\right)^{2}}{\sigma_{i}^{2}}\right)\right) M_{i}(\phi) d x+v \int\left|\nabla H_{\varepsilon}(\phi)\right| d x \\
& +E_{\text {boun }}+\lambda \int \frac{1}{2}(|\nabla \phi|-1)^{2} d x,
\end{aligned}
$$

where the first term is the image energy presented in [15], the second is the length term for smoothness [3], the third term is our novel boundary prior, and the final term is a signed distance regulariser to encourage the level set function to keep signed distance properties while it evolves [1]. $v$ and $\lambda$ are constants, which are set empirically. To get an accurate reading of the granule halo statistics, we need to exclude core pixels. The granule core is surrounded by the halo, therefore its intensities will be included when calculating the mean and variance within the contour. In order to account for this we determine the region statistical parameters in the following way: given a granule core, $C_{\text {core }}$, the mean and variance are determined for each region as follows:

$$
\left\{\begin{array}{l}
\mu_{i}(\phi)=\frac{\int I(x)\left(M_{i}(\phi(x)) \oplus C_{\text {core }}\right) d x}{\int\left(M_{i}(\phi(x)) \oplus C_{\text {core }}\right) d x} \\
\sigma_{i}^{2}(\phi)=\frac{\int\left(\mu_{i}-I(x)\right)^{2}\left(M_{i}(\phi(x)) \oplus C_{\text {core }}\right) d x}{\int\left(M_{i}(\phi(x)) \oplus C_{\text {core }}\right) d x}
\end{array}\right.
$$



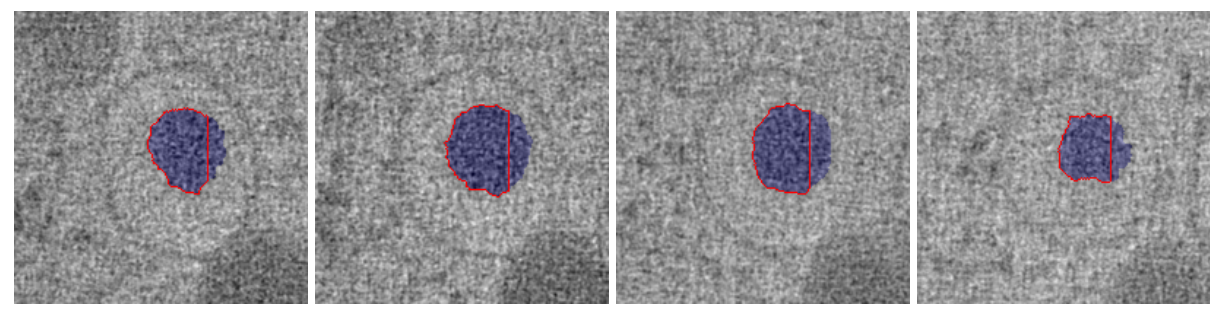

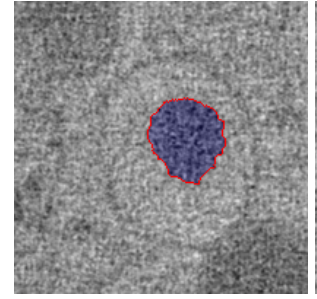

(a)

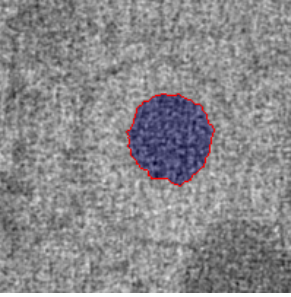

(b)

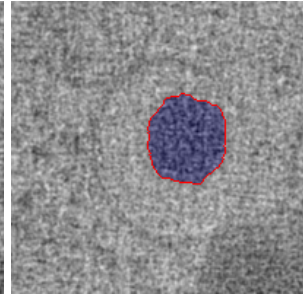

(c)

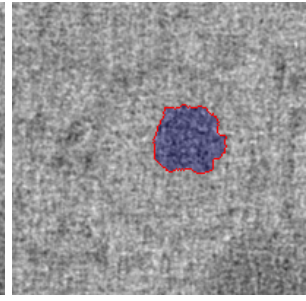

(d)

Figure 2: Effects of boundary prior term. Surface initialised within the core (top row). Slices of the surface are shown at $z=10,20,30$ and 40 in (a), (b), (c) and (d) respectively. The surface is shown after 10 iterations (bottom row). Note that the surface no longer is within the core.

To help prevent the contour from getting stuck in local minima during its evolution, we utilise the dual active contour framework [2.1]. Using the dual active contour, ultimately leads to a more accurate segmentation. The dual energy term is an internal energy, which means it is not influenced by image information. This results in an uniform attraction between the contours. This would be ideal if the granule membrane is always located in the centre of the two contour initialisations, however this is not always the case. Some granule membranes have an ellipsoid shape; also we do not know exactly how far away the membrane will be from the core. In some cases, granules have a membrane which is slightly visible. We introduce a gradient based term to help take advantage of this information and use it to slow the evolution of a contour around the granule membrane. The following gradient term is proposed,

$$
\begin{gathered}
g=\frac{1}{\left(1+\left|\nabla G_{\sigma_{s}} * I\right|^{4} C_{\text {coread } j}\right)} \\
C_{\text {coread } j}=1-\frac{G_{\sigma_{s}} * C_{\text {core }}}{\max \left(G_{\sigma_{s}} * C_{\text {core }}\right)} .
\end{gathered}
$$

Where $G_{\sigma_{s}}$ is a Gaussian smoothing kernel with standard deviation $\sigma_{s} . \mathrm{C}_{\text {coread } j}$ is included to help nullify any gradient information around the core. It should be noted that $\nabla G_{\sigma_{s}} * I$ is calculated only using $x$ and $y$ gradient information as in the $z$ dimension it does not provide any significant information. Using the fourth power of the image gradient is a reflection of the weak gradient information in the 3-D TEM images - we found that using this form provides sufficient results nonetheless. Given two contours initialised outside and inside the granule, with corresponding level set functions $\phi$ and $\psi$ respectively, we present our dual 


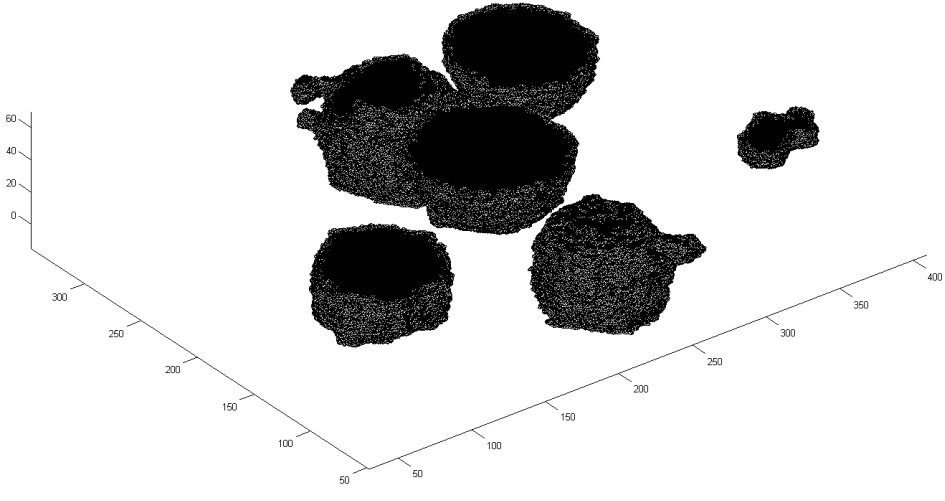

(a)

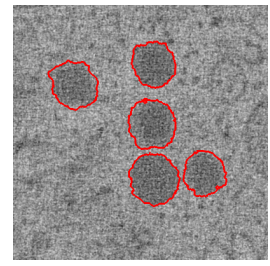

(b)

Figure 3: Core segmentation of section of image (a). Horizontal slice at $z=30$ with core overlaid in red (b).

energy functional as:

$$
E_{\text {dual }}(\phi, \psi)=E(\phi)+E(\psi)+\tau g \int\left[H_{\varepsilon}(\phi)+H_{\varepsilon}(\psi)\right]^{2} d x
$$

$E_{\text {dual }}$ can be minimised by the gradient descent method with respect to $\phi$ and $\psi$ by solving the following gradient flow equations:

$$
\begin{aligned}
& \frac{\partial \phi}{\partial t}=\delta_{\varepsilon}(\phi)\left(\log \left(\frac{\sigma_{2}^{2}}{\sigma_{1}^{2}}\right)-\frac{\left(I-\mu_{1}\right)^{2}}{\sigma_{1}^{2}}+\frac{\left(I-\mu_{2}\right)^{2}}{\sigma_{2}^{2}}\right)+v \delta_{\varepsilon}(\phi) \operatorname{div}\left(\frac{\nabla \phi}{|\nabla \phi|}\right) \\
& +\frac{\delta_{\varepsilon}(\phi) C_{\text {core }} \delta\left(\int\left(C_{\text {core }}-H_{\varepsilon}(\phi)\right) C_{\text {core }} d x\right)}{1-H\left(\int\left(C_{\text {core }}-H_{\varepsilon}(\phi)\right) C_{\text {core }} d x\right)}+\lambda\left(\nabla^{2} \phi-\operatorname{div}\left(\frac{\nabla \phi}{|\nabla \phi|}\right)\right) \\
& -2 \tau g \delta_{\varepsilon}(\phi)\left[H_{\varepsilon}(\phi)-H_{\varepsilon}(\psi)\right], \\
& \frac{\partial \psi}{\partial t}=\delta_{\varepsilon}(\psi)\left(\log \left(\frac{\sigma_{2}^{2}}{\sigma_{1}^{2}}\right)-\frac{\left(I-\mu_{1}\right)^{2}}{\sigma_{1}^{2}}+\frac{\left(I-\mu_{2}\right)^{2}}{\sigma_{2}^{2}}\right)+v \delta_{\varepsilon}(\psi) \operatorname{div}\left(\frac{\nabla \psi}{|\nabla \psi|}\right) \\
& +\frac{\delta_{\varepsilon}(\psi) C_{\text {core }} \delta\left(\int\left(C_{\text {core }}-H_{\varepsilon}(\psi)\right) C_{\text {core }} d x\right)}{1-H\left(\int\left(C_{\text {core }}-H_{\varepsilon}(\psi)\right) C_{\text {core }} d x\right)}+\lambda\left(\nabla^{2} \psi-\operatorname{div}\left(\frac{\nabla \psi}{|\nabla \psi|}\right)\right) \\
& -2 \tau g \delta_{\varepsilon}(\psi)\left[H_{\varepsilon}(\psi)-H_{\varepsilon}(\phi)\right] .
\end{aligned}
$$

Where $\partial \phi / \partial t$ and $\partial \psi / \partial t$ represent the Gâteaux derivatives of $E_{d u a l}$ with respect to $\phi$ and $\psi$, respectively. The order of the terms correspond to those in (5) and the final term is the dual term. $\tau$ is a weighting constant for the dual term and $\sigma_{1,2}$ is the standard deviation and $\mu_{1,2}$ the mean, for the corresponding level set functions.

\section{Segmentation Algorithm}

Insulin granule segmentation begins with core segmentation, it then uses the results of the core segmentation as a starting point for halo segmentation. We first do 2-D core segmenta- 
tion on each slice, to get an estimate of the core locations and shape. We utilise the regionbased active contour with shape regulariser in [13] to do the core segmentations. Due to the decreased detail in the 3-D TEM images, during the detection stage, granules may be missed in some slices. The advantage of doing the 2-D segmentation initially is that even if some granules are missed in a slice, it is likely that they will be segmented in others. Also, if there are false segmentations it is unlikely that they will persist through all slices. Due to the spherical shape of granules, it is likely that cores will be segmented within the middle than towards the ends. The roundness at the vertices is not captured by doing the 2-D segmentation, also it does not produce a smoothly connected core. In order to correct for this and encourage continuity, we extend 2-D dual region-based active contour in [13] to 3-D. Before we do active surface segmentation pre-processing is done to remove incorrect segmentations and connect disjoint cores. The result of this active surface is a smoother, more accurate, core segmentation. We then use the core as a starting point for halo segmentation. We utilise the dual active surface in (8). The core is used as the inner surface and a dilated version of the core is used at the outer surface. Pseudo-code of the granule segmentation algorithm is outlined in algorithm 1 .

Input: 3-D TEM Image $(I)$

$$
\text { /* 2-D Segmentation */; }
$$

\section{forall the Slices do}

Perform 2-D segmentation using [13];

end

$$
\text { /* Pre-processing */; }
$$

\section{forall the Connected components in I do}

I Remove objects with a thickness less than 5;

\section{end}

/*connect along z axis*/;

forall the Objects in I do

if (Object centres overlap in z axis) \& (Vertical distance $<\max$ (horizontal axis)) then

Connect Objects;

$$
\text { end }
$$

\section{end}

\section{forall the All granules do}

\section{I* 3-D Core Segmentation */;}

Crop area around granule to $2 \times \max$ (axis);

$\operatorname{adj}=(\max ($ Horizontal axis $)-$ Vertical axis $) \times 0.75$;

$m_{\text {outer }}=$ dilate $($ object, ones $[5,5, \operatorname{adj}+5])$;

$m_{\text {inner }}=\operatorname{erode}($ object, ones $[5,5, \operatorname{adj}-5])$;

$C_{\text {core }}=3$-D Dual Region-Based Active Surface;

$$
\text { / * 3-D Halo Segmentation */; }
$$

$m_{\text {outer }}=\operatorname{dilate}\left(C_{\text {core }}\right.$, ones $\left.[50,50,50]\right)$;

$m_{\text {inner }}=C_{\text {core }}$;

halo = Active Surface using (8); 


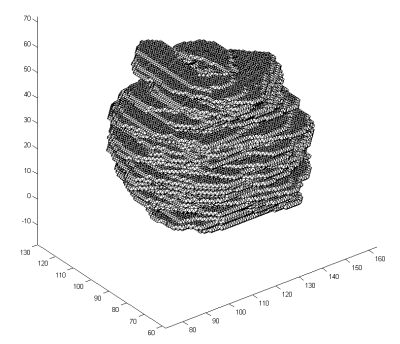

(a)

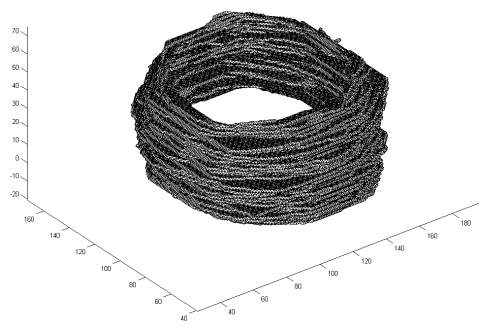

(c)

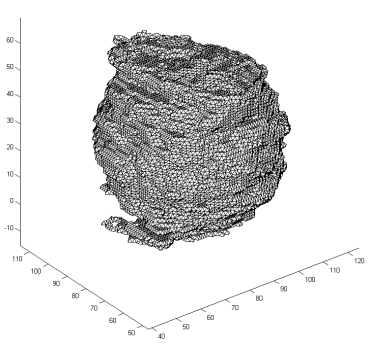

(b)

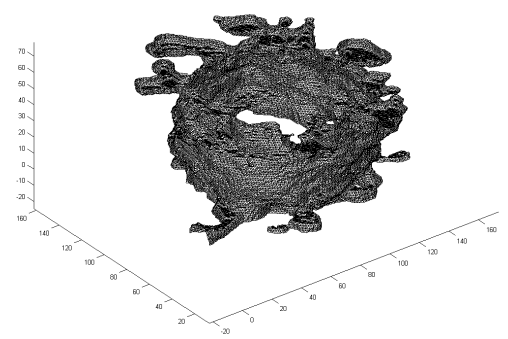

(d)

Figure 4: Core (a, b) and halo (c, d) segmentations done manually and using the proposed method in first and second columns respectively.

\begin{tabular}{cccccc}
\hline & \multicolumn{3}{c}{ Halo } & & Core \\
\cline { 2 - 4 } Method & Dual, [ij] & {$[15]$} & Proposed Method (8) & & Proposed Method \\
Dice's Coefficient & 0.82 & 0.49 & 0.85 & & 0.88 \\
\hline
\end{tabular}

Table 1: Core and halo segmentation comparisons against manual segmentations.

\section{Experimental Results and Analysis}

The 3-D TEM image used is $1000 \times 1000 \times 53$ pixels. For our novel active surface the following parameters are used for all images $v=0.009 \times 255^{2}, \lambda=0.000003 \times 255^{2}, \sigma_{s}=3.75$, $\varepsilon=0.005, \triangle t=0.2$ and $\tau=0.8160 \times 255^{2}$. To maintain a steady evolution, our boundary prior is normalised and then multiplied by 400 , (which was determined empirically). This is done because if the surface moves near the core, the surface is pushed out by a very large force - from our boundary term. Although conceptually correct, this greatly deforms the level set function (more than is necessary) leading to an unstable evolution. To demonstrate that our boundary prior is still effective with this scaling, in Fig. 2 we evolve a surface solely under the influence of our boundary term. The top row shows surface initialisations within the core (in blue) and after ten iterations we can see that the surface has moved outside of the core (top row). We perform core segmentation of a section of a 3-D TEM image to demonstrate the accuracy of the active surface used for core segmentation in, Fig. 3. Fig. 3 (a) shows the core segmentation — note the flat tops are at the image boundary — and Fig. 3 (b), shows a horizontal slice at $z=30$ with the core overlaid.

We show a completely encapsulated granule core and halo in Fig. 4. Fig. 4 (a and c) show a manually segmented core and halo, respectively. Note that since the granule is segmented 

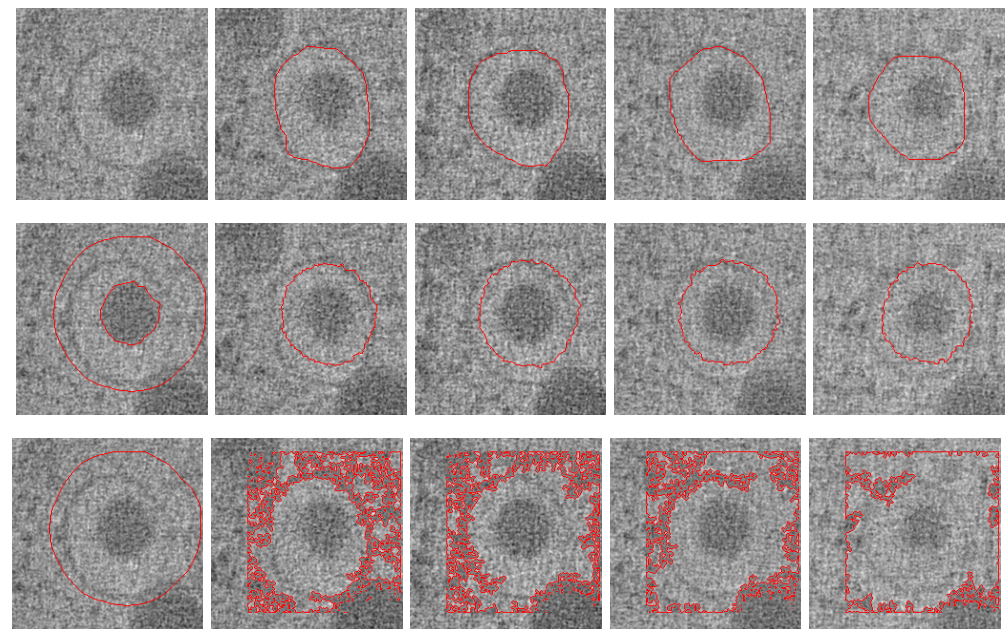

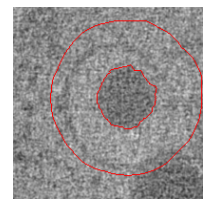

(a)

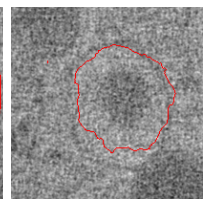

(b)

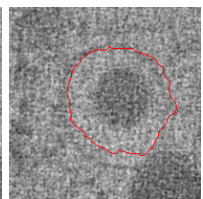

(c)

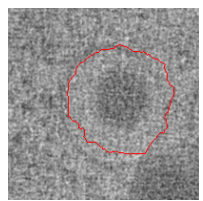

(d)

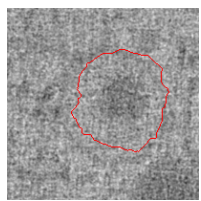

(e)

Figure 5: Halo segmentation comparisons: manual (first row), dual region-based active surface [I)] (second row), Rousson's [15] (third row) and proposed method (8) (bottom row).

in each slice, this does not ensure smoothness in the vertical plane. The segmented core and halo using our algorithm are shown in Fig. 4 (b and d), respectively. For the same granule we compare our active surface for halo segmentation against other active surfaces in Fig. 5. Results are shown at constant intervals, horizontal slices.

In Fig. 5 the results of halo segmentation are shown at slices 10, 20, 30 and 40 in the $z$ plane, (b), (c), (d) and (e) respectively. The contour initialisations are shown in (a) and are at $z=25$; the TEM image at that $z=25$ is shown at the top of (a). The first row in Fig. 5 shows the manually segmented results. In the second row, we show results using an active surface version of [1] in a dual framework. The third row is the result of [15]. The fourth row is the proposed active surface (8). All cases are initialised using our algorithm (with the exception of the third row, which is not dual). Where possible similar parameters are kept constant. We can see that our proposed active surface is able to achieve more accurate segmentations around the membrane, despite low contrast within the halo and a weak membrane. We also do a quantitative comparison of our method and the ones discussed previously, against the manually segmented granule, in Table 1 . We quantify our results using Dice's coefficient. It can be seen that the proposed active surface is able to achieve the most accurate segmentation around the membrane. 


\section{Conclusion}

We present an automated segmentation algorithm for insulin granule cores and halos, in 3D TEM beta cell images. The algorithm proceeds though 2-D core segmentation for core detections and then a more accurate core segmentation using a dual region-based active surface. To perform halo segmentation we introduce a dual Bayesian active surface. To slow contour attractions near the membrane, a gradient term is incorporated to take advantage of possible gradient information. We also propose a novel boundary prior to prevent the surface from moving into the core. We compare our novel active surface against other stateof-the-art active surface models. Qualitatively our method shows more accurate results. We also compare each method to a manually segmented granule using Dice's coefficient. Our method gives a coefficient of 0.88 and 0.85 for the core and halo respectively. Having a dedicated algorithm for insulin granule segmentation in 3-D TEM beta cell images will eliminate subjective bias and enable reproducibility. With this method researchers can acquire more accurate volumetric insulin granule information than previously possible.

\section{References}

[1] L. Aurélien, S. Kevin, A. Radhakrishna, K. Graham, and F. Pascal. Supervoxel-based segmentation of mitochondria in em image stacks with learned shape features. IEEE Trans. Med. Imag., 31(2):474-486, 2012.

[2] V. Caselles, R. Kimmel, and G. Sapiro. Geodesic active contours. Int. J. Comput. Vis., 22:61-79, 1995.

[3] T.F. Chan and L.A. Vese. Active contours without edges. IEEE Trans. Image Process., 10(2):266-277, Feb. 2001. ISSN 1057-7149. doi: 10.1109/83.902291.

[4] A. Dufour, V. Shinin, S. Tajbakhsh, N. Guillen-Aghion, J.-C. Olivo-Marin, and C. Zimmer. Segmenting and tracking fluorescent cells in dynamic 3-d microscopy with coupled active surfaces. IEEE Trans. Image Process., 14(9):1396-1410, Sept. 2005. ISSN 1057-7149. doi: 10.1109/TIP.2005.852790.

[5] O. Dzyubachyk, J. W. Niessen, and E. Meijering. Advanced level-set based multiplecell segmentation and tracking in time-lapse fluorescence microscopy images. in Proc. ISBI, pages 185-188, 2008.

[6] O. Dzyubachyk, W.A. van Cappellen, J. Essers, W.J. Niessen, and E. Meijering. Advanced level-set-based cell tracking in time-lapse fluorescence microscopy. IEEE Trans. Med. Imag., 29(3):852-867, March. 2010. ISSN 0278-0062. doi: 10.1109/ TMI.2009.2038693.

[7] E. Fava, J. Dehghany, J. Ouwendijk, A. Müller, A. Niederlein, P. Verkade, M. MeyerHermann, and M. Solimena. Novel standards in the measurement of rat insulin granules combining electron microscopy, high-content image analysis and in silico modelling. Diabetologia, 55:1013-1023, 2012. ISSN 0012-186X.

[8] L. Fuhui, P. Hanchuan, and M. Eugene. Automatic segmentation of nuclei in 3d microscopy images of c. elegans. in Proc. ISBI, pages 536-539, 2007. 
[9] R. Giuly, M. Martone, and M. Ellisman. Method: Automatic segmentation of mitochondria utilizing patch classification, contour pair classification, and automatically seeded level sets. BMC Bioinformatics, 13(1):29+, 2012. ISSN 1471-2105. doi: 10.1186/1471-2105-13-29.

[10] S. Göpel, Q. Zhang, L. Eliasson, X. Ma, J. Galvanovskis, T. Kanno, A. Salehi, and P. Rorsman. Capacitance measurements of exocytosis in mouse pancreatic alpha-, betaand delta-cells within intact islets of langerhans. J. Physiol., 556(3):711-726, 2004.

[11] J. Kremer. Computer visualization of three-dimensional image data using imod. $J$. Struct. Biol., 116(1):71-76, Jan. 1996. ISSN 10478477.

[12] C. Li, C.Y. Kao, J.C. Gore, and Z. Ding. Minimization of region-scalable fitting energy for image segmentation. IEEE Trans. Image Process., 17(10):1940-1949, Oct. 2008. ISSN 1057-7149.

[13] D. Nam, J. Mantell, D. Bull, P. Verkade, and A. Achim. Active contour based segmentation for electron microscopy images of insulin granule cores. in Proc. EMBC, Annual International Conference of the IEEE, San Diego, California, August. 2012.

[14] S. Osher and J.A. Sethian. Fronts propagating with curvature dependent speed: Algorithms based on hamilton-jacobi formulations. J. Comput. Phys., 79(1):12-49, 1988.

[15] M. Rousson and R. Deriche. A variational framework for active and adaptative segmentation of vector valued images. in Proc. Workshop Motion Vid. Computi., 2002.

[16] C. Siqi and R.J. Radke. Level set segmentation with both shape and intensity priors. in Proc. ICCV, pages 763-770, 2009. ISSN 1550-5499.

[17] S.G. Straub, G Shanmugam, and G.W.G. Sharp. Stimulation of insulin release by glucose is associated with an increase in the number of docked granules in the betacells of rat pancreatic islets. Diabetes, 53(12):3179-3183, 2004.

[18] A. Tsai, Jr. Yezzi, A., W. Wells, C. Tempany, D. Tucker, A. Fan, W.E. Grimson, and A. Willsky. A shape-based approach to the segmentation of medical imagery using level sets. IEEE Trans. Med. Imag., 22(2):137-154, 2003. ISSN 0278-0062.

[19] X. Wang and L. Wang. Color image segmentation based on bayesian framework and level set. in Proc. ICMLC, 6:3484-3489, 2008.

[20] X. Zhou and S.T.C. Wong. Informatics challenges of high-throughput microscopy. IEEE Signal Processing Mag., 23(3):63-72, May. 2006. ISSN 1053-5888.

[21] G. Zhu, Q. Zeng, and C. Wang. Dual geometric active contour for image segmentation. Opt. Eng., 45(8):080505-1-3, 2006. 